J. Clin. Chem. Clin. Biochem.

Vol. 27, 1989, pp. 445-449

(C) 1989 Walter de Gruyter \& Co.

Berlin - New York

\title{
Comparison of Direct Spectrophotometric Determinations of Bilirubin with Candidate Reference Method in Sera of Newborns
}

\author{
By $\leftrightarrows$. Hajzer \\ Oddelenie klinickej biochémie, Fakultná nemocnica s poliklinikou, Košice, ČSSR
}

(Received November 24, 1988/March 21, 1989)

Summary: Bilirubin was determined by direct spectrophotometry and by the candidate reference method of Doumas et al. (Clin. Chem. (1985) $31 ; 1789-1798)$ in a group of more than 230 newborns, and the results were compared. Direct spectrophotometry by Vink et al. (Clin. Chem. (1988) 34; 67-70) gives the following regression equation in comparison with the reference method: $y=0.967 x+6.57$ with $r=0.9874$. Comparison of direct spectrophotometry at 463 and $582 \mathrm{~nm}$ (for the simultaneous determination bilirubin and $\mathrm{HbO}_{2}$ ) with the reference method gave the following regression equation: $y=0.971 x+8.16$ with $r=0.9814$. Two methods of direct two-wavelength spectrophotometry showed statistically insignificant differences, using significance levels $p=0.05$ and $p=0.01$, in comparison with the reference method. In our group of newborns we determined serum $\mathrm{HbO}_{2}$ concentrations from $0.2 \mathrm{~g} / 1$ (as a 0.025 fractile) to $2.6 \mathrm{~g} / 1$ (as a 0.975 fractile) with a median value of $0.76 \mathrm{~g} / \mathrm{l}$. In a micromodification, the reference method is insensitive to $\mathrm{HbO}_{2}$ in concentrations of up to $5 \mathrm{~g} / \mathrm{l}$. Both direct spectrophotometric methods are insensitive to $\mathrm{HbO}_{2}$ concentrations up to $25 \mathrm{~g} / \mathrm{l}$. Direct spectrophotometric methods give results identical to those of the reference method and therefore are very suitable for use in neonatology.

\section{Introduction}

The use of the caffeine reagent in the direct spectrophotometry of bilirubin was introduced in 1986 by Vink et al. The most significant property of this reagent is the independence of the molar absorptivity of bilirubin of the serum protein matrix (1). This independence allows the use of bilirubin standards with various human or bovine albumins as a protein base (2). Vink et al. proposed in 1988 the use of the caffeine reagent in the direct spectrophotometry of unconjugated bilirubin in sera of newborns. According to Vink's study, only one correction is necessary in this method, i. e. the correction for $\mathrm{HbO}_{2}$ by measurement at 465 and $528 \mathrm{~nm}$. Using a small group of 55 specially selected sera of newborns, the results of direct spectrophotometry were compared with those of the candidate reference method of Doumas et al. These results indicate that the Doumas' method is reliable only for minimal haemolysis when the concentration of $\mathrm{HbO}_{2}$ is approximately $0.6 \mathrm{~g} / 1$ (3). This finding is not in agreement with the results of Doumas et al. (4).

Since we considered the given group of newborns to be too small for such a comparison, and because the candidate reference method is insensitive to $\mathrm{HbO}_{2}$ up to $2 \mathrm{~g} / 1$ (4), we verified the direct spectrophotometric method on more than 230 newborns. In all tested sera we determined the $\mathrm{HbO}_{2}$ concentration, because its interference is still a subject of discussion (3). From the absorption spectrum of $\mathrm{HbO}_{2}$ in the caffeine reagent, several pairs of wavelengths can be found for which the equation $A_{\lambda 1}=A_{\lambda 2}$ is valid. For the simultaneous determination of $\mathrm{HbO}_{2}$ and bilirubin in the same reaction mixture we introduced another pair of wavelengths, namely 463 and $582 \mathrm{~nm}$. The first wavelenght is situated near the maximum of bilirubin absorption $(457 \mathrm{~nm})$, the second one near the maximum of $\mathrm{HbO}_{2}$ absorption ( $576 \mathrm{~nm}$ ). Moreover, absorbance of bilirubin at $582 \mathrm{~nm}$ is negligible. 
In our study we compared the results from both direct spectrophotometric methods with those from the candidate reference method of Doumas et al. (4) using linear regression analysis and a paired t-test of statistical significance. By means of statistical methods we evaluated the $\mathrm{HbO}_{2}$ concentration levels in the group of our newborns and its effect upon the result of analysis.

\section{Materials and Methods}

\section{Apparatus}

The absorption spectra of $\mathrm{HbO}_{2}$ and bilirubin in the caffeine reagent was measured on a Varian DMS 100 UV-VIS spectrophotometer (Varian Associates Ltd., England). The spectral bandwidth was $1.0 \mathrm{~nm}$. The accuracy of the wavelength was checked by using the deuterium lamp at 486 and $656.1 \mathrm{~nm}$ as the reference.

In all other measurements a routine digital spectrophotometer SPEKOL 11 (VEB Carl Zeiss Jena, G.D.R.) was used. The accuracy of the photometer response was tested at $401 \mathrm{~nm}$ using alkaline 4-nitrophenol according to the method of Bowers \& $M c C o m b$ (5). The spectral bandwidth of this equipment is $11 \mathrm{~nm}$. The pathlength of the spectrophotometric cuvettes was $10.02 \mathrm{~mm}$.

\section{Dispensor}

Dispensette ${ }^{\otimes}$ type Variable (Brand, Wertheim, F.R. G.) 0.4$2.0 \mathrm{ml}$. Reproducibility for the volume of $1.0 \mathrm{ml}$ was within $0.2 \%$ and was tested by weighing at $20^{\circ} \mathrm{C}$.

\section{Pipettes}

Transferpette ${ }^{\circledR}$-piston pipettes for fixed volume of $20 \mu \mathrm{l}$ and $50 \mu \mathrm{l}$ (Brand, Wertheim, F. R. G.). Reproducibility was within $0.4 \%$ and $0.2 \%$, respectively; accuracy was better than $0.7 \%$ and $0.5 \%$, respectively. Glass pipettes were tested by weighing the delivered volume at $20^{\circ} \mathrm{C}$, and accepted if the measured volume differed from the nominal value by less than $0.2 \%$.

\section{Chemicals}

All chemicals used for the preparation of reagents were analytical grade. Double distilled water from glass apparatus was used for the preparation of solutions. All chemicals were supplied by Lachema Corp. Brno, Czechoslovakia.

\section{Bilirubin standard}

We used a commercially produced freeze-dried standard of bilirubin supplied as Bio-LA-Test ${ }^{\star}$ BILIRUBIN STANDARD kit by Lachema Corp. Brno, Czechoslovakia. This standard is supplied in brown vials under reduced pressure and it contains bovine serum albumin as a protein base. The bilirubin content of this product was $355 \mu \mathrm{mol} / \mathrm{l}$. Its spectrophotometric properties are described below (see Results).

\section{Sera from newborns}

The group of newborns was not specially selected according to the previously given criteria. Newborns were younger than seven days post partum. All blood samples were obtained by venapuncture. The blood was collected, shielded from light, and centrifuged immediately after clotting. Sera were analysed immediately or stored at $-20^{\circ} \mathrm{C}$ until the day of analyses. Sera were stored no longer than seven days. All analyses were made in duplicate.

\section{The modifications of compared methods}

\section{Diazo method}

All reagents were prepared as described by Doumas et al. (4). We used his candidate reference method in the modification for a small volume of serum.

Procedure: Pipette $0.8 \mathrm{ml}$ of caffeine reagent and $0.02 \mathrm{ml}$ of sample (serum, standard, etc.), mix and let stand for 10 minutes. Then add $0.2 \mathrm{ml}$ of working diazo reagent, mix immediately and let stand at room temperature for 10 minutes. Add $0.6 \mathrm{ml}$ of alkaline tartrate solution to the reaction mixture and wait for 10 minutes before reading the absorbance. The sample blank was prepared by the same procedure but sulphanilic acid was substituted for diazo reagent. The absorbance was measured at $598 \mathrm{~nm}$. The volume fraction of serum in the whole reaction mixture was 0.012 while in the original Doumas' method this fraction is $\mathbf{0 . 0 5 9}$. This modification is suitable for the measurement of bilirubin in sera of neonates, because a smaller volume of analysed sample is required and the sensitivity of the deter: mination is acceptable. Moreover, interference from $\mathrm{HbO}_{2}$ and other substances possibly present in the reaction mixture is lowered.

\section{Caffeine method}

We used the caffeine method described by Vink et al. The caffeine reagent was prepared as described in l.c. (1).

The working procedure was modified as follows: Pipette 0.05 $\mathrm{ml}$ of sample (serum, standard, etc.) and add $1.0 \mathrm{ml}$ of caffeine reagent. After about 10 minutes read the absorbance of ths mixture against the caffeine reagent. The absorbances were measured at two pairs of wavelengths. The first two wavelengths ( 465 and $528 \mathrm{~nm}$ ) were those used by Vink et al.; the second two (463 and $582 \mathrm{~nm}$ ) were chosen to enable the simultaneous determination of $\mathrm{HbO}_{2}$ in the tested sera.

\section{Calculations}

Bilirubin concentration was calculated according to equation 2 published in the study of Vink et al. (3).

$$
\begin{aligned}
& \mathrm{C}_{\mathrm{UB}}=\frac{\left(\mathrm{A}_{465}-\mathrm{A}_{528}\right)_{\text {test }}}{\left(\mathrm{A}_{465}-\mathrm{A}_{528}\right)_{\text {stand }}} \times c_{\text {standard, }} \mu \mathrm{mol} / 1 \\
& \mathrm{C}_{\mathrm{UB}}=\frac{\left(\mathrm{A}_{463}-\mathrm{A}_{582}\right)_{\text {cest }}}{\left(\overline{\mathrm{A}}_{463}-\mathrm{A}_{582}\right)_{\text {stand }}} \times c_{\text {standard, }} \mu \mathrm{mol} / 1
\end{aligned}
$$

Sera were analysed in duplicate and the bilirubin standards in five parallel analyses. In further mathematical evaluation the mean values of the calculated concentrations were used.

\section{Results}

\section{Bilirubin standard properties}

The calibration material contained $355 \mu \mathrm{mol} / \mathrm{l}$ of unconjugated bilirubin in bovine serum albumin. It was prepared so that the concentration of bovine serum albumin was $40 \mathrm{~g} / 1$. This calibrator has similar spectral properties to those described by Vink et al. (1) (fig. 1). We calculated the values of molar absorptivity for 


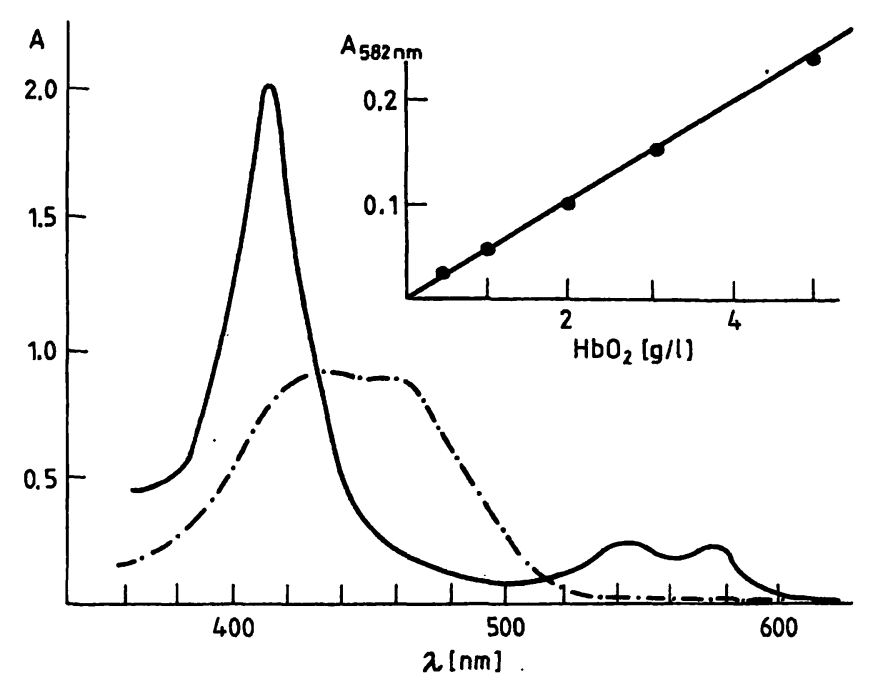

Fig. 1. Absorbance spectra for $\mathrm{HbO}_{2}$ and bilirubin standard in caffeine reagent.

- spectrum of $\mathrm{HbO}_{2}$

-.-..- $\quad$ spectrum of bilirubin standard used (concentration $355 \mu \mathrm{mol} / \mathrm{l}$ )

Upper corner: Absorbance-concentration relationship of $\mathrm{HbO}_{2}$ standards at $582 \mathrm{~nm}$ in caffeine reagent.

both absorption maxima from the declared concentration of bilirubin. The molar absorptivities of unconjugated bilirubin at $432 \mathrm{~nm}$ and $457 \mathrm{~nm}$ were $4896.2 \mathrm{~m}^{2} \cdot \mathrm{mol}^{-1}$ and $4890.7 \mathrm{~m}^{2} \cdot \mathrm{mol}^{-1}$, respectively. These values differed by about $-0.7 \%$ and $+0.4 \%$, respectively, from the published ones (1).

\section{$\mathrm{HbO}_{2}$ concentrations in sera from newborns}

The $\mathrm{HbO}_{2}$ concentration was calculated from the absorbance at $582 \mathrm{~nm}$. The calibration function was determined by a set of $\mathrm{HbO}_{2}$ standards. Figure 1 shows the calibration function of $\mathrm{HbO}_{2}$ at $582 \mathrm{~nm}$ in caffeine reagent. The range of $\mathrm{HbO}_{2}$ values was determined by means of a non-parametric statistic test as the 0.025 and 0.975 fractiles for a group of 236 newborns. The determined $\mathrm{HbO}_{2}$ concentrations ranged between $0.2 \mathrm{~g} / 1$ and $2.6 \mathrm{~g} / 1$ with a median value of $0.76 \mathrm{~g} / 1$. The frequency distribution of $\mathrm{HbO}_{2}$ in sera of our groüp is shown in figure 2.

\section{Effect of $\mathrm{HbO}_{2}$}

We investigated the effect of $\mathrm{HbO}_{2}$ on the results of compared methods by addition of haemolysate to the pooled serum of newborns. We verified this effect in the concentration range from $0.5 \mathrm{~g} / 1$ to $25 \mathrm{~g} / 1 \mathrm{HbO}_{2}$. The results of these observations, which are summa-

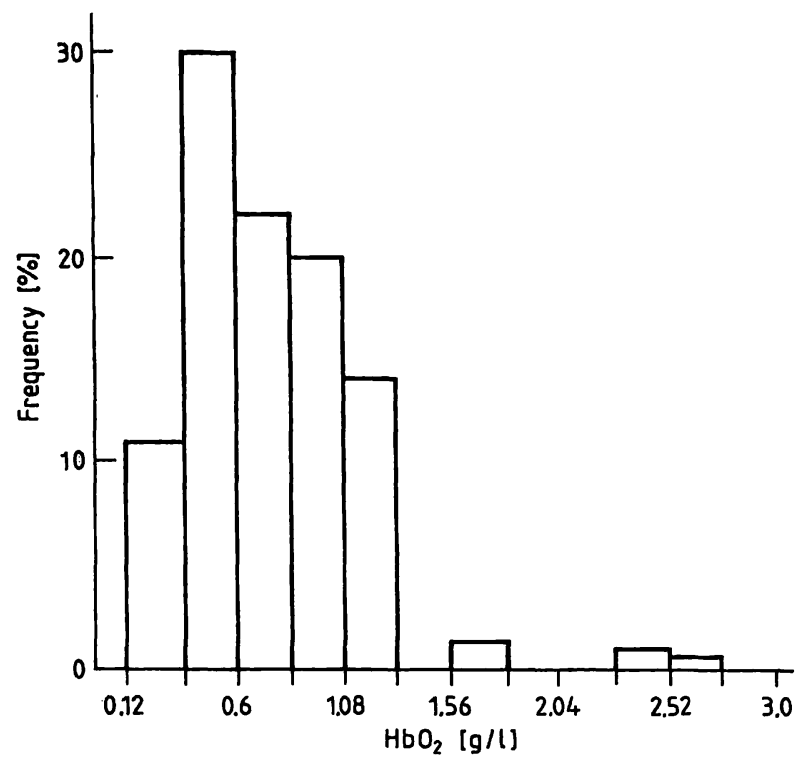

Fig. 2. Frequency distribution of serum $\mathrm{HbO}_{2}$ values in sera of 236 neonates.

rized in table 1 , show that the micromodification of the candidate reference method of Doumas et al. is insensitive to $\mathrm{HbO}_{2}$ up to $5 \mathrm{~g} / \mathrm{l}$. Measurement of absorbance at two different wavelengths by direct spectrophotometry can be used to correct completely for the influence of $\mathrm{HbO}_{2}$ up to $25 \mathrm{~g} / \mathrm{l}$.

Tab. 1. Effect of $\mathrm{HbO}_{2}$ on bilirubin* results of compared methods.

\begin{tabular}{|c|c|c|c|c|}
\hline \multicolumn{2}{|c|}{$\mathrm{HbO}_{2}$ concentration } & \multicolumn{3}{|c|}{$\%$ of original bilirubin measured } \\
\hline \multirow[t]{2}{*}{$\overline{\mathrm{g} / 1}$} & \multirow[t]{2}{*}{$\mu \mathrm{mol} / 1$} & \multirow{2}{*}{$\begin{array}{l}\text { Doumas } \\
\text { et al. }\end{array}$} & \multicolumn{2}{|c|}{ Direct spectrophotometry } \\
\hline & & & Vink et al. & $463 / 582 \mathrm{~nm}$ \\
\hline 0 & 0 & 100.0 & 100.0 & 100.0 \\
\hline 0.5 & 31 & 99.9 & 99.0 & 99.8 \\
\hline 1.0 & 62 & 100.2 & 98.8 & 99.9 \\
\hline 1.5 & 93 & 100.1 & 98.4 & 99.6 \\
\hline 2.0 & 124 & 99.6 & 98.2 & 99.6 \\
\hline 2.5 & 155 & 101.2 & 99.8 & 101.6 \\
\hline 5.0 & 310 & 100.9 & 98.6 & 100.0 \\
\hline 10.0 & 620 & 90.4 & 98.0 & 99.6 \\
\hline 15.0 & 930 & 85.8 & 96.8 & 100.5 \\
\hline 20.0 & 1240 & 80.8 & 97.3 & 101.5 \\
\hline 25.0 & 1550 & 69.0 & 99.8 & 97.8 \\
\hline
\end{tabular}

* concentration of bilirubin was $236 \mu \mathrm{mol} / 1$ in pooled sera of newborns

\section{Results of linear regression analysis}

We evaluated all results of bilirubin determinations by means of linear regression analysis. The results of two-wavelength spectrophotometry at 463 and 582 $\mathrm{nm}$ were compared with those from the candidate reference method. The equation of the regression line for a group of 236 newborns is $y=0.971 x+8.16$ with correlation coefficient $r=0.9814$ (fig. 3 ). 


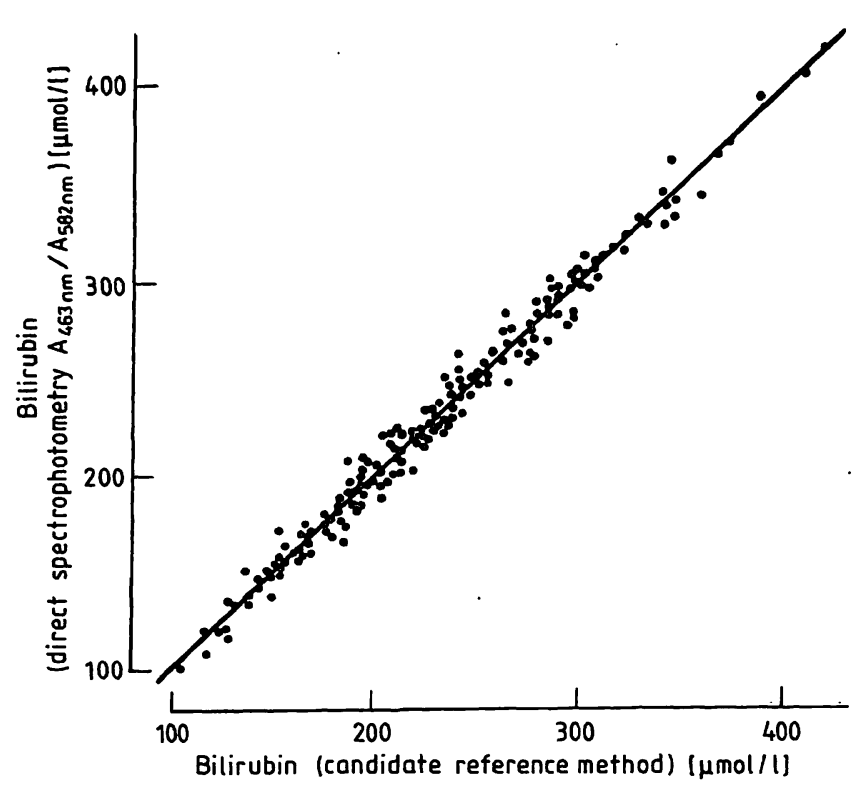

Fig. 3. Comparison of the direct spectrophotometry at 463 and $582 \mathrm{~nm}$, resp. (y) with the candidate reference method of Doumas et al. (x).

Regression line: Correlation coefficient Number of samples:

$$
\begin{aligned}
& y=0.971 x+8.16 \\
& r=0.9814 \\
& n=236
\end{aligned}
$$

The regression equation $y=0.967 x+6.57$ with correlation coefficient $\mathrm{r}=\mathbf{0 . 9 8 7 4}$ was obtained for a group of 103 newborns. This equation is valid for the comparison of two-wavelength spectrophotometry at 465 and $528 \mathrm{~nm}$ (according to Vink et al.) with the candidate reference method of Doumas et al. (fig. 4).

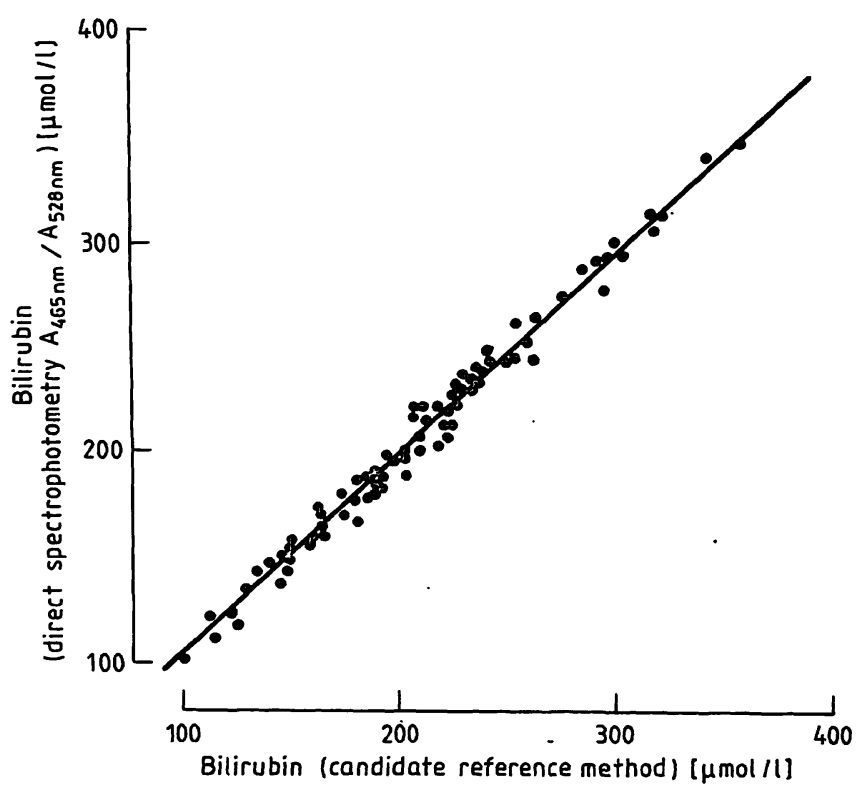

Fig. 4. Comparison of the direct spectrophotometry of bilirubin according to Vink et al. (y) with the candidate reference method of Doumas et al. (x).

Regression line:

Correlation coefficient:

$y=0.967 x+6.57$

Number of samples:

$\mathrm{r}=0.9874$

$\mathrm{n}=103$

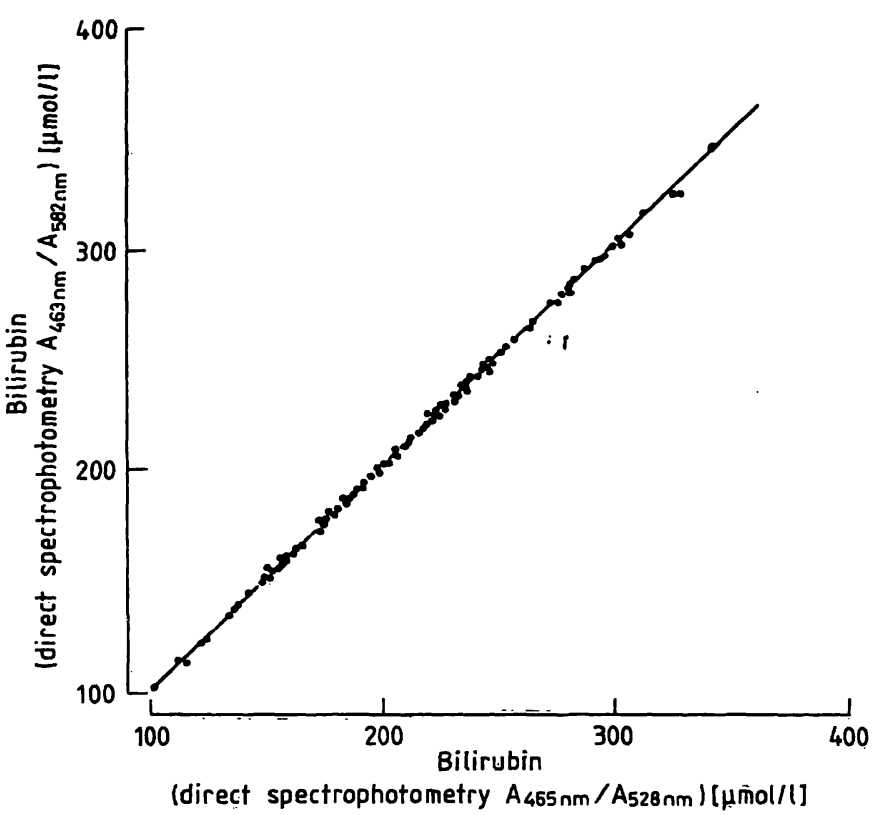

Fig. 5. Comparison of the both methods of direct spectrophotometry. Measuring of absorbance at 465 and $528 \mathrm{~nm}$ (Vink et al.) (x); measuring of absorbance at 463 and $582 \mathrm{~nm}(\mathrm{y})$.

Regression line:

Correlation coefficient:

Number of samples:

$$
\begin{aligned}
& y=1.004 x+1.03 \\
& r=0.9994 \\
& n=103
\end{aligned}
$$

The comparison of two different pairs of wavelengths (463 and $582 \mathrm{~nm}$ versus 465 and $528 \mathrm{~nm}$ ) for twowavelength direct spectrophotometry is illustrated on figure 5. The equation of regression line is $y=1.004 \mathrm{x}$ +1.03 with correlation coefficient $r=0.9994$, which confirms the identity of the results obtained by spectrophotometry at either pair of wavelengths.

\section{Discussion}

The aim of the present study was:

- to compare the results of direct spectrophotometry with those from the candidate reference method on a large group of newborns.

- to re-evaluate the interference of haemolysis and its influence on the reliability of the results obtained by the direct two-wavelength spectrophotometry and by the method of Doumas et al.

- to verify the practical possibility of using both analytical principles interchangeably in daily routine practice.

The results of our study can be summarized as follows.

1. In the comparison of direct spectrophotometry in caffeine reagent with the candidate reference method by Doumas we did not obtain the excellent correlation coefficients reported by Vink et al. (3). The correlation coefficients recently published by Vink et al. were 
$\mathrm{r}=0.9997, \mathrm{r}=0.9986$ and $\mathrm{r}=0.9978$ for groups of $\mathrm{n}=24, \mathrm{n}=18$ and $\mathrm{n}=13$ newborns, respectively. The correlation coefficients for our group of 103 and 236 newborns were $r=0.9874$ and $r=0.9814$, respectively. Unfortunately, we could not examine the same number of samples for both comparisons, because the volume of blood was insufficient. The discrepancy might be explained by the larger number of samples and the heterogeneity of the compared groups, as well as by some medicaments used in the treatment of newborns. However, the statistical evaluation showed no significant differences between the results of direct spectrophotometry and those from the candidate reference method, using significance levels $p=0.05$ and $p=0.01$. Therefore, we consider that the two analytical principles give identical results.

2. The effect of haemolysis was described in detail by Doumas et al. (4) and Vink et al. (3). The results of our study confirm the findings of Doumas et al. that the candidate reference method is insensitive to $\mathrm{HbO}_{2}$ up to $2 \mathrm{~g} / \mathrm{l}$. This method, in a described micromodification, is insensitive to $\mathrm{HbO}_{2}$ up to $5 \mathrm{~g} / \mathrm{l}$. Direct twowavelength spectrophotometry in caffeine reagent is insensitive to $\mathrm{HbO}_{2}$ up to $25 \mathrm{~g} / \mathrm{l}$ (tab. 1).

\section{References}

1. Vink, K. L. J., Schuurman, W. \& van Gansewinkel, R. (1986) Use of the Caffeine Reagent in Direct Spectrophotometry of Bilirubin. Clin. Chem. 32, 1389-1393.

2. Vink, K. L. J., van Dreumel, R. J., Schuurman, W., Wikkeling, H., van Gansewinkel, R., Phielix, C. J. \& Koedam, H. C. (1987) A Candidate Standard for Use in Calibration of Total Bilirubin in Serum. Clin. Chem. 33, 1817-1821.

3. Vink, K. L. J., Schuurman, W. \& van Gansewinkel, R. (1988) Direct Spectrophotometry of Bilirubin in Serum of the Newborn, with Use of Caffeine Reagent. Clin. Chem. 34, 67-70.
3. We used direct spectrophotometry at 463 and 582 $\mathrm{nm}$ for the simultaneous determination of $\mathrm{HbO}_{2}$. The aim was to verify the $\mathrm{HbO}_{2}$ concentration in the sera of newborns from the absorbance value at $582 \mathrm{~nm}$. This determination appeared to be less sensitive than measuring at the absorption maximum, but quite sufficient for the present purpose. The $\mathrm{HbO}_{2}$ concentration in more than $90 \%$ of newborns from our group is lower than $1.32 \mathrm{~g} / 1$ (fig. 2). Some extremely high values of $\mathrm{HbO}_{2}$ might be explained, for example, by incorrect venapuncture. From a practical point of view, haemolysis represents no risk of erroneous results in the majority of samples, either by direct spectrophotometry or by the method of Doumas.

From all these findings it can be concluded that direct spectrophotometry is very suitable for the examination of sera from newborns; it is simple, rapid, and adequately reliable. Therefore, we prefer this method for routine neonatal diagnosis.

\section{Acknowledgement}

We are grateful to Mrs. Eve Rakoczi for skilful technical assistance.

4. Doumas, B. T., Kwok-Cheung, P. P., Perry, B. W., Jendrzejczak, B., McComb, R. B., Schaffer, R. \& Hause, L. L. (1985) Candidate Reference Method for Determination of Total Bilirubin in Serum: Development and Validation. Clin. Chem. 31, 1779-1789.

5. Bowers, G. N. \& McComb, R. B. (1975) Measurement of Total Alkaline Phosphatase Activity in Human Serum. Clin. Chem. 21, 1988-1995.

\section{Dr. Štefan Hajzer}

Oddelenie klinickej biochémie

Fakultná nemocnica s poliklinikou

Rastislavova 43

ČS-041 90 Košice 
